$\xi=-1$

\title{
Active Noise Cancellation for Predefined Voices
}

\author{
D. Venkata Ram Reddy ${ }^{1 *}$, A.V. Prabhu ${ }^{2 *}$, M. Sri Krishna Chaitanya ${ }^{3 *}$, Gopiram.k ${ }^{4 *}$ \\ ${ }^{1,3}$ U.G Students, Dept. of E.C.M Koneru Lakshmaiah Education Foundation, Vaddeswaram, Guntur, Andhra Pradesh, India \\ ${ }^{2}$ Dept. of E.C.M Koneru Lakshmaiah Education Foundation, Vaddeswaram, Guntur, Andhra Pradesh, India -522502 \\ ${ }^{4}$ Dept. of E.C.E Koneru Lakshmaiah Education Foundation, Vaddeswaram, Guntur, Andhra Pradesh, India -522502 \\ *Email: ramud52@gmail.com
}

\begin{abstract}
This paper describes the Active noise cancellation for predefined voice. It is another technique of cancelling the noise with adaptive filter. Its Pros lies in this, with no apriority evaluations of noise, we can decrease the noise reduction, or we can try to decrease the strength by designing the filters. If we can't find the frequency of the signal, we have to design fixed filter. It wants two inputs one is primary input which contain voice and some noise and a reference input containing noise. And the second input is the reference input which is filtered, and we can hear it with noise reduction and we can see it signal representation. Active filtering before subtraction allows the treatment of data sources that square measure settled or arbitrary, stationary or time-variable. Further this project can be developed by taking the two voices at a time and we can hear a single voice which is filtered.
\end{abstract}

\section{Introduction}

In analog signal there will be an error which is corrupted by many ways, where in communication noise will be an error. When the signal is converted from electrical energy to electromagnetic energy. In nature there are some sources where it may affect the electromagnetic energy, by this the error will occur which mean an unwanted signal has been added to the main signal, to remove this unwanted signal there are many ways to remove it but, in some cases, the main signal is also removed while the unwanted signals are removed. To remove this unwanted signal, we can use adaptive filtering using LMS (Least mean squares). Hearing loss is one among the foremost current chronic health conditions, touching concerning five hundred million individuals world-wide. In keeping with several surveys, one out of 10 individuals suffer from hearing disorder and would get pleasure from exploitation hearing aids. Owing to the accumulated exposure to noise in standard of living and therefore the aging of the world's population, this variety is predicted to any increase within the future. The foremost common kind of disability is sensorineural hearing disorder. Individuals with this sort of hearing disorder don't solely suffer from associate degree accumulated hearing threshold however conjointly from reduced speech discrimination ability, creating it tough for them to speak in strident environments. Compensation of sensorineural hearing disorder doesn't solely need amplification of the perceived sound signal, as is completed in ancient hearing aids, however conjointly reduction of the ground noise with relevancy the specified speech signal. Recent advances in physical science shrinking created it potential to integrate a digital signal processor and 2 (or more) microphones in business hearing instruments. However, thanks to the dearth of effective digital signal process algorithms, the extra potentialities of digital signal

process isn't however absolutely exploited, so the advantage of business hearing aids for hearing impaired individuals remains restricted. Business hearing aids don't sufficiently discriminate between speech and noise.

Moreover, in real-life use, they usually suffer from an extra performance loss thanks to imperfections like electro-acoustic transducer match, so intelligibility in noise is hardly or insufficiently improved. Additionally, as hearing aids become smaller and smaller, acoustic feedback, i.e. the physics coupling between the loudspeaker system and therefore the microphone(s) of the hearing aid, poses a serious downside to hearing aid users. Acoustic feedback ends up in severe distortion of the predetermined noise and wailing if the portable hearing assistant pick up is gathered. As a result, the most amplification that may be utilized in an advertisement hearing aid is commonly too tiny to catch up on the hearing disorder during a patient. Therefore, associate degree pressing demand exists for economical and well working signal process algorithms for noise reduction and acoustic feedback suppression. Headphones that utilize active noise cancellation apply completely different techniques like adaptive filtering. Active noise cancellation sees to invert the signal that deconstructivity, close noise. The disadvantage of active noise cancellation is that the compromise created in audio quality yet because the value. Whether or not the method is completed hardware or software package with a DSP chip, the noise created, and primary input voice can share identical frequency content, leading to cancellation of each.

\section{System Model}

\section{Gaussian Noise:}

It defines the measurable noise having a likelihood distribution work which is equivalent to ordinary distribution is known as Gaussian noise. 


$$
p(x)=\frac{1}{\sigma \sqrt{2 \pi}} e^{\frac{-(x-\mu)^{2}}{2 \sigma^{2}}}
$$

Where $\mathrm{z}$ indicates dark level, $\mu$ shows mean value and sigma demonstrates the standard deviation

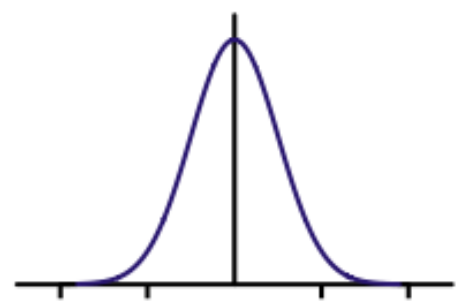

Fig 1: Shape of the impulse response of a typical Gaussian Noise

Syntax for Gaussian noise in MATLAB:

$\mathrm{y}=\operatorname{awgn}(\mathrm{x}, \mathrm{snr})$

$\mathrm{y}=\operatorname{awgn}(\mathrm{x}, \mathrm{snr}$, sigpower $)$

$\mathrm{y}=\operatorname{awgn}\left(\mathrm{x}, \mathrm{snr}\right.$, 'measured' $^{\prime}$

$\mathrm{y}=\operatorname{awgn}($, streamhandle $)$

$\mathrm{y}=\operatorname{awgn}($, seed $)$

$\mathrm{y}=\operatorname{awgn}(\ldots$, powertype $)$

where $y=\operatorname{awgn}(\mathrm{x}, \mathrm{snr})$ adds the Gaussian noise to vector scale signal $\mathrm{x}$. snr represents signal to noise ratio in DB.awgn adds complex noise. $y=a w g n(x, s n r, s i g p o w e r)$ represents input power in db. $y=\operatorname{awgn}(x, s n r, ' m e a s u r e d ')$ represents the power measured before adding noise. $\mathrm{y}=\mathrm{awgn}\left(\_,\right.$stream handle) explains about repeatable noise samples with using function of reset.y=awgn(),seed) it explains about seed value initiated for a signal before adding of the noise. $y=a w g n($,powertype) it explains about the signal power in terms of $\mathrm{dB}$ and linear.

Adaptive Filtering:

The structure that characterizes how the yield signal of the channel is figured from its information signal can be said as adaptive filtering. The parameters with in the structure can be iteratively changed to alter the filters input and output relations. It describes the adjustment of the parameters from instant time to the next. The normal adaptive filter application is to utilize a filter which identify the unknown system, for example, the response of unknown signal transmission, to pick genuinely different applications like echo cancellation and channel ID.

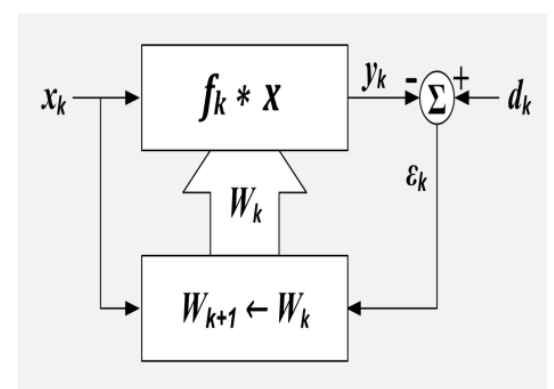

Fig 2: Structure of Adaptive Filtering

Where $\mathrm{k}$ indicates sample number, $\mathrm{x}$ demonstrates reference input, $\mathrm{X}$ is set of late estimations of $\mathrm{x}, \mathrm{d}$ is desired input, $\mathrm{W}$ isset of channel coefficients, $\varepsilon$ is error output, $f$ is filter response reaction, $\Sigma$ shows summation, upper box is linear filter, bring down box is adaption algorithm.

\section{LMS Filter:}

Least mean squares calculations of a class of versatile channel used to copy a coveted channel by finding the channel coefficients that identify with creating the minimum mean square of the blunder flag. It is a stochastic inclination drop strategy in that channel is just adjusted in view of the blunder at present time.

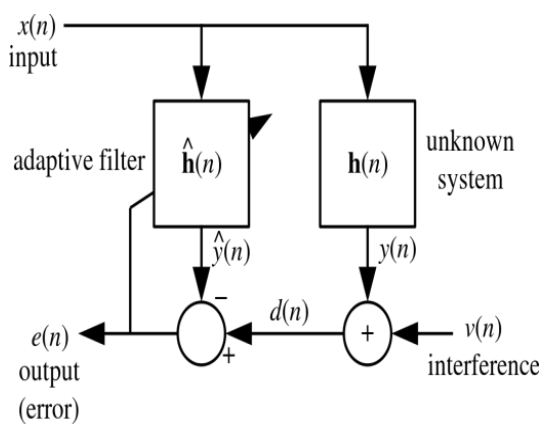

Fig 3: LMS (Least Mean Square) working

From the above Fig 1, Fig 2and Fig 3 we can design the Fig 4 (system model). The working process of the system model can be briefly explained in proposed system

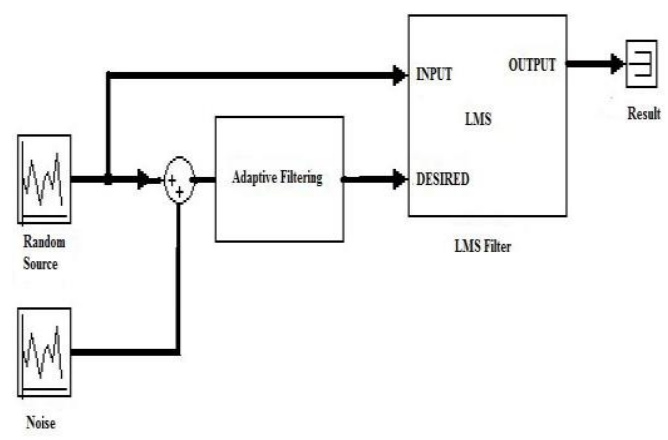

Fig 4: Block representation for Active Noise Cancellation for Predefined Voices

\section{Proposed System}

The system needs two inputs primary input which consist of random source voices like human voice, Music, industrial sounds etc., which will be recorded by a microphone and later we convert the fil into .WAV file which stands for wave form audio file this file helps to compute in Mat lab with help of GUI interface Graphical user interface. And now we use a second input named as reference input which consist of extra noise the noise may be Gaussian noise or white noise and now we can hear the combination of reference input and primary input in headphones which not filtered or where noise cancellation does not take place. For cancelling the noise, we have used the LMS algorithm where the summation of two inputs goes to the adaptive filtering and we can get a desired output now plating the wave of primary input, reference input and desired output which can be seen in fig 6 . And later in LMS algorithm we can smooth the signal using SavitzkyGolay smoothing filter and finally the result wav can be saved with the help of GUI.

\section{Existing Technology}

Previously they have designed the noise cancellation in passive where they optimize the background noise with a fixed feedback controller performs the compensation task. They used the methods of optimization from control theory which is in terms of filter [1]. While to cancel the noise we have used the Concepts of LMS 
algorithm and adaptive filtering where they are in the existing technology we have added an update to them is giving extra noise like Gaussian noise and white noise [2]. The cancellation in Bluetooth stereo headphones they have used the broadband feedback control, narrowband feedforward control and adaptive feedback control are used from these technologies we have adopted the adaptive filtering concepts so that which helps the cancellation of both primary input and reference input [3].

\section{Discussion of Results}

For Brief Explanation we are taking a small example to measure a noise in the room. At first, we measure the primary input voice in terms of $\mathrm{dB}$ (Decibels). Let us assume the voice be $50 \mathrm{Db}$ and we are taking the external noise be $30 \mathrm{~dB}$. Now the summing of both primary input voice and the external noise the total decibels are $80 \mathrm{~dB}$. Now with the help of microphone the external noise is taken and then inverted to cancel the external noise from the summing noise. The noise is cancelled at $40 \mathrm{db}$. Then the desired output voice is $40 \mathrm{db}$ is plotted in amplitude and magnitude waveform and saved as file name.wav

The Figure 5 Explains the essential information voice given by Human or music which is recorded by means of amplifier and it is spared in the organization of. Wav(Waveform Audio File arrange) MATLAB will plot the greatness and stage in the recurrence space and in addition the waveform in the time area. We can see the commotion exhibit in the wave frame with the assistance of antinodes and hubs.

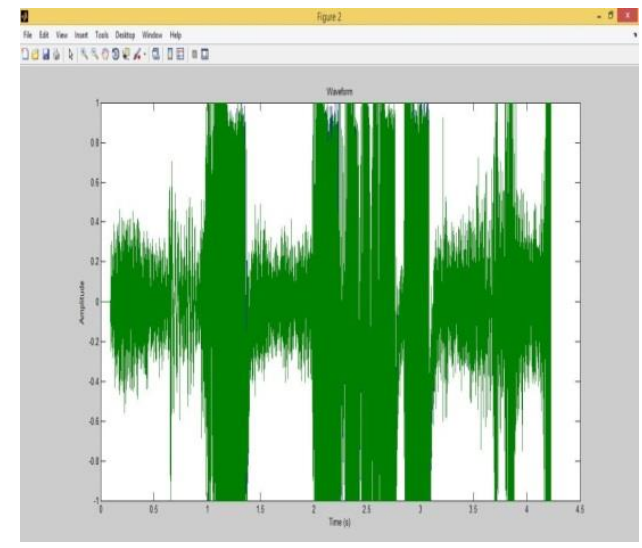

Fig 5: Primary voice input

The Figure 6 clarifies the essential information voice, noise created through Gaussian channel and last commotion crossed out wave at exact of $85 \%$. At first we can hear the essential information voice and later we can hear the additional noise with essential info voice to get the commotion crossed out wave. We have to reverse the additional commotion motion by knowing the parameters of created Noise.

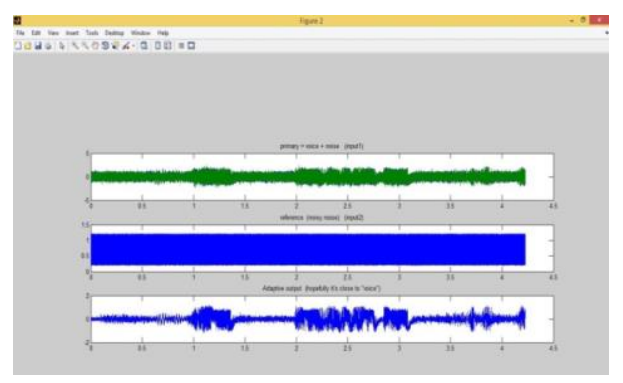

Fig 6: Noise cancellation wave

\section{Conclusion}

Active Noise Cancelling for Predefined voices is another manner of cancelling noise from the signal. The benefit of the signal is its less output noise, and less frequency. The dynamic capacity allows the procedure of sources of info whose properties are obscure and at times non-stationary. Square measure typically achieved with typical adaptive channel setups. This Project shows the enormous choice of utilization during where Active Noise Cancelling is utilized. The Experimental outcomes demonstrate the advantages of active noise cancellation for predefined voices. In each example dropping was refined with little signal distortion regardless of the way that the frequencies of the signal and impedance covered. Subsequently it sets up the nature of active noise cancellation procedures and its different applications [5].

\section{Future Work}

As of now we have taken only one reference input to cancel noise in it by adding knowing fixed noises like Gaussian noise or white noise so that we can know the frequencies in the noise and later inverting the signal we can cancel the noise in both reference input and added noise. The future work can develop by taking two reference input and design of fixed filter in our own standards. For designing a filter, we must and should know the noise frequency in the signal. And the second development is taking two reference input at a time using GUI interface in MATLAB so that after cancelling noise we can hear only one voice from it.

\section{References}

[1] Active noise cancellation in headphones by digital robust feedback control Stefan Liebich; Carlotta Anemuller; Peter Vary; Peter Jax; Daniel Ruschen ; Steffen Leonhardt , Year: 2016.

[2] Prediction Filter Design for active noise cancellation Headphones Markus Guldenschuh; Robert Holdrich, IET Signal Processing. Year: 2013.

[3] Review of DSP algorithms for active noise control S.M.Kuo; $\underline{\text { D. }}$ R. Morgan, Proceedings of the 2000. IEEE International Conference on Control Applications. Conference Proceedings, Year: 2000.

[4] B. Widrow, er al., "Adaptive Noise cancelling: Principles and Applications", Proc. IEEE, vol. 63, pp.1692-1716, Dec. 1975.

[5] Alias Free Sub band Adaptive Filtering With Critical Sampling, SangGyun Kim; Chang D. Yoo; Truong Q. Nguyen, IEEE Transactions on Signal Processing, Year: 2008.

[6] John R. Glover, Jr., "Adaptive Noise Cancelling Applied to curving Interferences", IEEE Trans. ASSP, Vol. ASSP-25, No. 6, pp. 484-491, Dec. 1977.

[7] J.R. Zeidler et al., "Adaptive improvement of Multiple Sinusoids in Noise", IEEE Trans. ASSP, Vol. ASSP-26, No. 3, pp. 240-254, June 1978 .

[8] D. W. Tufts, "Adaptive Line improvement and Spectrum Analysis", Proc. IEEE (Letts.), vol. 65, pp.169-170, Jan. 1977.

[9] S. Kuo and D. Morgan, "Active noise control: a tutorial review," Proceedings of the IEEE, Jun 1999.

[10] M. Bai and D. Lee, "Implementation of an active headset by using the H robust control theory," Acoustical Society of America Journal, Oct. 1997.

[11] S. Skogestad and I. Postlethwaite, "Multivariable feedback control: analysis and design". John Wiley \& Sons, 2005.

[12]Xiang, N., Schroeder, M.R.: "Reciprocal maximum-length sequence pairs for acoustical dual source measurements". J. Acoust. Soc. Am., Year :2003.

[13] Akhtar, M.T., Abe, M., Kawamata, M.: 'A new variable step size LMS algorithm-based method for improved online secondary path modelling in active noise control systems', IEEE Trans. Audio, Speech, Lang. Process., Year: 2006 